\title{
The development and validation of a scoring tool to predict the operative duration of elective laparoscopic cholecystectomy
}

\author{
Reshma Bharamgoudar ${ }^{1}$. Aniket Sonsale ${ }^{1}$. James Hodson ${ }^{2}$. Ewen Griffiths ${ }^{3,4}$. on behalf of the CholeS Study \\ Group, West Midlands Research Collaborative
}

Received: 2 September 2017 / Accepted: 3 January 2018 / Published online: 16 January 2018

(c) The Author(s) 2018. This article is an open access publication

\begin{abstract}
Background The ability to accurately predict operative duration has the potential to optimise theatre efficiency and utilisation, thus reducing costs and increasing staff and patient satisfaction. With laparoscopic cholecystectomy being one of the most commonly performed procedures worldwide, a tool to predict operative duration could be extremely beneficial to healthcare organisations.

Methods Data collected from the CholeS study on patients undergoing cholecystectomy in UK and Irish hospitals between 04/2014 and 05/2014 were used to study operative duration. A multivariable binary logistic regression model was produced in order to identify significant independent predictors of long (>90 min) operations. The resulting model was converted to a risk score, which was subsequently validated on second cohort of patients using ROC curves.

Results After exclusions, data were available for 7227 patients in the derivation (CholeS) cohort. The median operative duration was $60 \mathrm{~min}$ (interquartile range $45-85$ ), with $17.7 \%$ of operations lasting longer than $90 \mathrm{~min}$. Ten factors were found to be significant independent predictors of operative durations $>90 \mathrm{~min}$, including ASA, age, previous surgical admissions, BMI, gallbladder wall thickness and CBD diameter. A risk score was then produced from these factors, and applied to a cohort of 2405 patients from a tertiary centre for external validation. This returned an area under the ROC curve of 0.708 $(\mathrm{SE}=0.013, p<0.001$ ), with the proportions of operations lasting $>90 \mathrm{~min}$ increasing more than eightfold from 5.1 to $41.8 \%$ in the extremes of the score.

Conclusion The scoring tool produced in this study was found to be significantly predictive of long operative durations on validation in an external cohort. As such, the tool may have the potential to enable organisations to better organise theatre lists and deliver greater efficiencies in care.
\end{abstract}

Keywords Laparoscopic cholecystectomy · Patient factors · Operative duration · Scoring tool · Prediction · Theatre utilisation

Ewen Griffiths

ewen.griffiths@uhb.nhs.uk

1 College of Medical \& Dental Sciences, University of Birmingham, Birmingham, UK

2 Institute of Translational Medicine, University Hospitals Birmingham NHS Foundation Trust, Birmingham, UK

3 Institute of Cancer and Genomic Sciences, College of Medical and Dental Sciences, University of Birmingham, Birmingham, UK

4 Department of Upper Gastrointestinal Surgery, University Hospitals Birmingham NHS Foundation Trust, Birmingham, UK
There are 70,000 cholecystectomies performed in the UK each year, making it one of the most common general surgical operations [1]. The average operative duration for this laparoscopic procedure is usually $<1 \mathrm{~h}$ [2]. With the average hourly cost for an operating theatre being $£ 1200$, efforts to utilise every minute of allocated theatre time is vital [3]. This is especially so in a resource constrained National Health Service (NHS) working environment that is required to save $£ 20$ bn by 2020 to remain sustainable for the future [4]. Poor planning can lead to cancellations, which are expensive for the Trust and, more importantly, distressing for patients. One particular study found that $63 \%$ of on-the-day cancellations were due to a lack of theatre time [5], with another study demonstrating that approximately 
$30 \%$ of lists are under-run, leaving the operating theatre idle [6]. Careful planning and scheduling is therefore paramount to increase operating theatre efficiency and, in doing so, it is estimated that NHS trusts can make efficiency savings of approximately $£ 4 \mathrm{~m}$ per year [3]. In a time where rising demographic pressures are demanding ever increasing spending on healthcare, it is vital we optimise the use of our existing resources.

Previous research in other surgical areas has assessed a variety of patient factors and their impact on operative duration [7-11], but studies in laparoscopic cholecystectomy are few and a predictive scoring tool is yet to be developed. Traditionally, surgeons have estimated their operative durations, but research has demonstrated that these estimates are often inaccurate [6]. Many hospitals have now moved to electronic systems and central schedulers with the aim of reducing costs. However, such systems require significant learning time to adapt to organisational needs [12]. Historical procedure and surgeon data have been used to estimate operative duration. However, this is known to be of low accuracy, as it fails to account for pre-operative patient factors [12].

We aimed to create a clinically useful scoring tool to predict the operative duration of laparoscopic cholecystectomy using pre-operative patient factors and to externally validate its reliability using a separate dataset.

\section{Methods}

Two datasets were used in this study, to allow for a risk score to be produced and externally validated:

\section{CholeS dataset}

The CholeS study was a multicentre, prospective population-based cohort study that assessed variations in patient factors with outcomes of cholecystectomy [13, 14]. The protocol for this study has been published previously [15]. Data were collected from 8820 patients who underwent a laparoscopic cholecystectomy at 166 hospitals in the UK between March to April 2014, and was found to be $99.2 \%$ accurate by independent data validation. Pre-operative variables included patient demographics, indications for surgery, admission type, ASA grade, ultrasound findings and preoperative ERCP. Surgical duration was calculated from the time (minutes) of skin incision to end of skin closure.

For this study, all patients undergoing emergency cholecystectomy were excluded $(N=1420)$, as these are associated with long procedure times and elective theatre utilisation was the focus of this study. In addition, those where the operative duration was not recorded $(N=168)$ were excluded, as were those with an operative duration $<10 \mathrm{~min}$
$(N=5)$, as these were thought to be unrealistic. This left a total of 7227 patients for analysis.

\section{Validation dataset}

The validation dataset was retrospectively collected from the University Hospitals Birmingham NHS Foundation Trust (UHB) - a large tertiary hospital with 1213 inpatient beds and 32 operating theatres [16]. Data were collected for all elective laparoscopic cholecystectomies carried out between 2010 and 2016, excluding emergencies, as well as those having combined procedures. This left a total of $N=2405$ patients for analysis. Data for surgical duration and preoperative factors were gathered from a variety of electronic hospital systems, including the Lorenzo patient information system, Galaxy operating theatre system and PICS (Prescribing Information and Communication system). ASA grades were calculated using comorbidities derived from the informatics database, and were based on the definitions provided by the American Society of Anaesthesiologists [17].

\section{Statistical methods}

Initially, the operative duration was dichotomised into groups of $\leq 90$ vs. $>90 \mathrm{~min}$. Univariable analyses were then performed, comparing the rates of operative durations $>90$ min across a range of factors. Comparisons across categorical factors were made using Fisher's exact tests, whilst Mann-Whitney tests were used to compare ordinal factors between the operative duration groups.

A multivariable analysis was then performed to identify independent predictors of operative duration. A binary logistic regression model was produced, with a forwards stepwise approach used to select variables for inclusion. The resulting model was then converted to a risk score, by rounding the beta coefficient (log-odds) of each factor to the nearest 0.5 , after multiplying by a constant to minimise the impact of rounding errors. Where this resulted in negative values, the reference category was changed, such that all values in the score were positive. The predictive accuracy of the model was then assessed using ROC curves. The model was also applied to a second cohort of patients for external validation.

All analyses were performed using IBM SPSS 22 (IBM Corp. Armonk, NY), with $p<0.05$ deemed to be indicative of statistical significance throughout.

\section{Results}

\section{Demographics}

After exclusions, data were available for 7227 surgeries in the derivation (CholeS) cohort. The patients had a mean 
Table 1 Univariable analysis of associations between operative duration and both demographic and pre-operative factors

\begin{tabular}{|c|c|c|c|}
\hline & $N$ & $\begin{array}{l}\text { Operative } \\
\text { dura- } \\
\text { tion }>90 \mathrm{~min}\end{array}$ & $p$ Value \\
\hline Age (years) & & & $<0.001 * *$ \\
\hline$<30$ & 854 & $90(10.5 \%)$ & \\
\hline $30-39$ & 1035 & $128(12.4 \%)$ & \\
\hline $40-49$ & 1397 & $237(17.0 \%)$ & \\
\hline $50-59$ & 1534 & $297(19.4 \%)$ & \\
\hline $60-69$ & 1381 & $282(20.4 \%)$ & \\
\hline $70+$ & 1023 & $245(23.9 \%)$ & \\
\hline Gender & & & $<0.001$ \\
\hline Female & 5406 & $852(15.8 \%)$ & \\
\hline Male & 1821 & $427(23.4 \%)$ & \\
\hline Indication for surgery & & & $<0.001$ \\
\hline Acalculous/cholecystitis & 1739 & $483(27.8 \%)$ & \\
\hline CBD stone & 479 & $145(30.3 \%)$ & \\
\hline Colic/dyskinesia/polyp & 4435 & $541(12.2 \%)$ & \\
\hline Pancreatitis & 570 & $109(19.1 \%)$ & \\
\hline BMI & & & $<0.001 *$ \\
\hline$<25$ & 1475 & $219(14.8 \%)$ & \\
\hline $25-30$ & 2465 & $424(17.2 \%)$ & \\
\hline $31-35$ & 1689 & $305(18.1 \%)$ & \\
\hline$>35$ & 1324 & $279(21.1 \%)$ & \\
\hline CBD diameter & & & $<\mathbf{0 . 0 0 1}$ \\
\hline Normal & 6013 & $936(15.6 \%)$ & \\
\hline Dilated & 1063 & $310(29.2 \%)$ & \\
\hline Gallbladder wall & & & $<0.001$ \\
\hline Normal & 5017 & $708(14.1 \%)$ & \\
\hline Thick walled & 2053 & $530(25.8 \%)$ & \\
\hline Pre-operative MRCP & & & $<0.001$ \\
\hline No & 5325 & $857(16.1 \%)$ & \\
\hline Yes & 1819 & $419(23.0 \%)$ & \\
\hline Pre-operative CT & & & $<\mathbf{0 . 0 0 1}$ \\
\hline No & 6158 & $1006(16.3 \%)$ & \\
\hline Yes & 978 & $271(27.7 \%)$ & \\
\hline Pre-operative ERCP & & & $<0.001$ \\
\hline No & 6349 & $1049(16.5 \%)$ & \\
\hline Yes & 783 & $227(29.0 \%)$ & \\
\hline Grade of senior surgeon & & & $0.005^{*}$ \\
\hline$<$ ST5 & 308 & $42(13.6 \%)$ & \\
\hline$>$ ST6 & 1165 & $183(15.7 \%)$ & \\
\hline Consultant & 5748 & $1052(18.3 \%)$ & \\
\hline Planned intra-op cholangiogram & & & $<0.001$ \\
\hline No & 6519 & $1070(16.4 \%)$ & \\
\hline Yes & 655 & $203(31.0 \%)$ & \\
\hline $\begin{array}{l}\text { Number of previous surgical } \\
\text { admissions }\end{array}$ & & & $<\mathbf{0 . 0 0 1} *$ \\
\hline 0 & 4006 & $535(13.4 \%)$ & \\
\hline 1 & 2424 & $546(22.5 \%)$ & \\
\hline 2 & 486 & $118(24.3 \%)$ & \\
\hline
\end{tabular}

Table 1 (continued)

\begin{tabular}{lccc}
\hline & $N$ & $\begin{array}{l}\text { Operative } \\
\text { dura- } \\
\text { tion }>90 \mathrm{~min}\end{array}$ & $p$ Value \\
\hline$>2$ & 170 & $57(33.5 \%)$ & \\
ASA & & & $<\mathbf{0 . 0 0 1} *$ \\
1 & 2803 & $352(12.6 \%)$ & \\
2 & 3687 & $722(19.6 \%)$ & \\
$>2$ & 690 & $199(28.8 \%)$ & \\
\hline
\end{tabular}

Data reported as $N(\%)$, with $p$ values from Fisher's exact tests, unless stated otherwise

* $p$ Value from a Mann-Whitney test, to account for the ordinal nature of the factor

**p Value from a Mann-Whitney test, using the exact age. Bold $p$ values are significant at $p<0.05$

age of 51 years $(\mathrm{SD}=16)$, and the majority were female (74.8\%). The median operative duration was $60 \mathrm{~min}$, with an interquartile range (IQR) of 45-85 min. Operations lasted for $>90 \mathrm{~min}$ in $17.7 \%(N=1279)$ of the cohort.

\section{Risk score derivation}

Associations between the operative duration and a range of demographic and pre-operative factors are reported in Table 1. All of the factors considered were found to be significantly associated with longer operative durations. For this reason, a multivariable analysis was performed, in order to identify which factors were independently associated with operative duration (Table 2). This analysis found the likelihood of an operation taking $>90 \mathrm{~min}$ to increase significantly with BMI $(p<0.001)$, ASA grade $(p<0.001)$, and the number of previous surgical admissions that the patient had $(p=0.005)$. In addition, the indication on admission significantly influenced operative duration $(p<0.001)$, with patients admitted with acalculous and cholecystitis being the most likely to have operations lasting $>90 \mathrm{~min}$. Patients with a thickened gallbladder, a dilated CBD diameter, or who had received a pre-operative CT or planned intra-operative cholangiogram were significantly more likely to take $>90$ min (all $p<0.001)$. In addition, patients of male gender $(p=0.002)$ and aged $40+(p=0.004)$ were also at significantly higher risk of requiring a longer operative duration.

These 10 factors were then combined to form a risk score (Table 3). This score has a potential range from 0 to 20 , although the observed range in the cohort was $0.5-17.5$ (median $=5$ ). A ROC curve analysis returned an area under the curve (AUROC) of $0.696(\mathrm{SE}=0.009, p<0.001)$ for the prediction of operations lasting $>90 \mathrm{~min}$. 
Table 2 Multivariable analysis of predictors of $>90 \mathrm{~min}$ operations

\begin{tabular}{|c|c|c|c|}
\hline & Beta $^{a}$ & Odds ratio $(95 \% \mathrm{CI})$ & $p$ Value \\
\hline Age (years) & & & 0.004 \\
\hline$<30$ & 0 & 1 & - \\
\hline $30-39$ & 0.058 & $1.06(0.78-1.45)$ & 0.714 \\
\hline $40-49$ & 0.430 & $1.54(1.16-2.05)$ & 0.003 \\
\hline $50-59$ & 0.438 & $1.55(1.17-2.06)$ & 0.002 \\
\hline $60-69$ & 0.366 & $1.44(1.07-1.93)$ & 0.015 \\
\hline $70+$ & 0.380 & $1.46(1.07-2.00)$ & 0.017 \\
\hline Gender (male) & 0.241 & $1.27(1.09-1.48)$ & 0.002 \\
\hline Indication & & & $<0.001$ \\
\hline Acalculous/cholecystitis & 0 & 1 & - \\
\hline CBD stone & -0.154 & $0.86(0.66-1.12)$ & 0.258 \\
\hline Colic/dyskinesia/polyp & -0.527 & $0.59(0.49-0.71)$ & $<0.001$ \\
\hline Pancreatitis & -0.627 & $0.53(0.41-0.70)$ & $<0.001$ \\
\hline BMI & & & $<0.001$ \\
\hline$<25$ & 0 & 1 & - \\
\hline $25-30$ & 0.208 & $1.23(1.01-1.49)$ & 0.035 \\
\hline $31-35$ & 0.291 & $1.34(1.09-1.65)$ & 0.006 \\
\hline$>35$ & 0.532 & $1.70(1.36-2.13)$ & $<0.001$ \\
\hline CBD diameter (dilated) & 0.535 & $1.71(1.42-2.05)$ & $<0.001$ \\
\hline Gallbladder wall (thick) & 0.371 & $1.45(1.24-1.70)$ & $<0.001$ \\
\hline Pre-operative CT & 0.320 & $1.38(1.15-1.65)$ & $<0.001$ \\
\hline Planned intra-op cholangiogram & 0.706 & $2.03(1.66-2.47)$ & $<0.001$ \\
\hline Number of previous surgical admissions & & & 0.005 \\
\hline 0 & 0 & 1 & - \\
\hline 1 & 0.202 & $1.22(1.03-1.45)$ & 0.020 \\
\hline 2 & 0.227 & $1.25(0.96-1.64)$ & 0.095 \\
\hline$>2$ & 0.630 & $1.88(1.29-2.74)$ & 0.001 \\
\hline ASA & & & $<0.001$ \\
\hline 1 & 0 & 1 & - \\
\hline 2 & 0.225 & $1.25(1.06-1.47)$ & 0.007 \\
\hline$>2$ & 0.630 & $1.88(1.48-2.39)$ & $<0.001$ \\
\hline
\end{tabular}

Results are from a multivariable binary logistic regression model with a forward stepwise approach to variable selection. All factors from Table 1 were considered for inclusion in the model. Bold $p$ values are significant at $p<0.05$

${ }^{\text {a }}$ The beta coefficients (i.e. log-odds) from the model

\section{Validation}

The risk score was then applied to a cohort of $N=2405$ patients from UHB for external validation. This cohort had a similar operative duration to the derivation cohort, with a median of 66 min (IQR: 52-85), and with $20.0 \%(N=481)$ of operations taking $>90 \mathrm{~min}$. The median risk score was found to be 4.5 , with a range from 0 to 14.5 . The score as a whole had a similar degree of predictive accuracy to that observed in the derivation cohort, with an AUROC of 0.708 $(\mathrm{SE}=0.013, p<0.001)($ Fig. 1$)$.

\section{Discussion}

Our study has created a scoring tool that uses pre-operative patient factors to predict the probability that a laparoscopic cholecystectomy will take $>90 \mathrm{~min}$. This scoring tool has also been successfully externally validated against a separate dataset and has demonstrated predictive accuracy. The results showed that, for low-scoring individuals vs. highscoring individuals, the proportion of operations taking $>90$ min increases significantly from 5.8 to $41.4 \%$.

We hope this scoring tool could enable better planning and utilisation of elective theatre lists. A better 
Table 3 Risk score

\begin{tabular}{|c|c|}
\hline & Points \\
\hline \multicolumn{2}{|l|}{ Age (years) } \\
\hline$<40$ & 0 \\
\hline $40+$ & 1.5 \\
\hline \multicolumn{2}{|l|}{ Gender (male) } \\
\hline Female & 0 \\
\hline Male & 1 \\
\hline \multicolumn{2}{|l|}{ Indication } \\
\hline Pancreatitis & 0 \\
\hline Colic/dyskinesia/polyp & 0.5 \\
\hline CBD stone & 2 \\
\hline Acalculous/cholecystitis & 2.5 \\
\hline \multicolumn{2}{|l|}{ BMI } \\
\hline$<25$ & 0 \\
\hline $25-35$ & 1 \\
\hline$>35$ & 2 \\
\hline \multicolumn{2}{|l|}{ CBD diameter } \\
\hline Normal & 0 \\
\hline Dilated & 2 \\
\hline \multicolumn{2}{|l|}{ Gallbladder wall } \\
\hline Normal & 0 \\
\hline Thick & 1.5 \\
\hline \multicolumn{2}{|l|}{ Pre-operative CT } \\
\hline No & 0 \\
\hline Yes & 1.5 \\
\hline \multicolumn{2}{|c|}{ Planned intra-op cholangiogram } \\
\hline No & 0 \\
\hline Yes & 3 \\
\hline \multicolumn{2}{|c|}{ Number of previous surgical admissions } \\
\hline 0 & 0 \\
\hline $1-2$ & 1 \\
\hline$>2$ & 2.5 \\
\hline \multicolumn{2}{|l|}{ ASA } \\
\hline 1 & 0 \\
\hline 2 & 1 \\
\hline$>2$ & 2.5 \\
\hline
\end{tabular}

Based on the multivariable analysis in Table 2 . The number of points for each factor was calculated by rounding the beta coefficient to the nearest 0.5 , after multiplying by 4 to minimise rounding errors. Categories for a factor that had the same number of points (e.g. age $<30$ and 30-39) were combined to simplify the table

understanding of patient factors that result in increased operative duration and how this affects surgeon workload can help to optimise theatre scheduling and result in fewer cancellations. With theatres being one of the most expensive resources to run [18], even small improvements in theatre utilisation have the potential to impact costs greatly, and with many trusts under pressure to tackle unsustainable deficits [19], it is possible that utilising this scoring tool may be helpful in addressing this issue. This is particularly

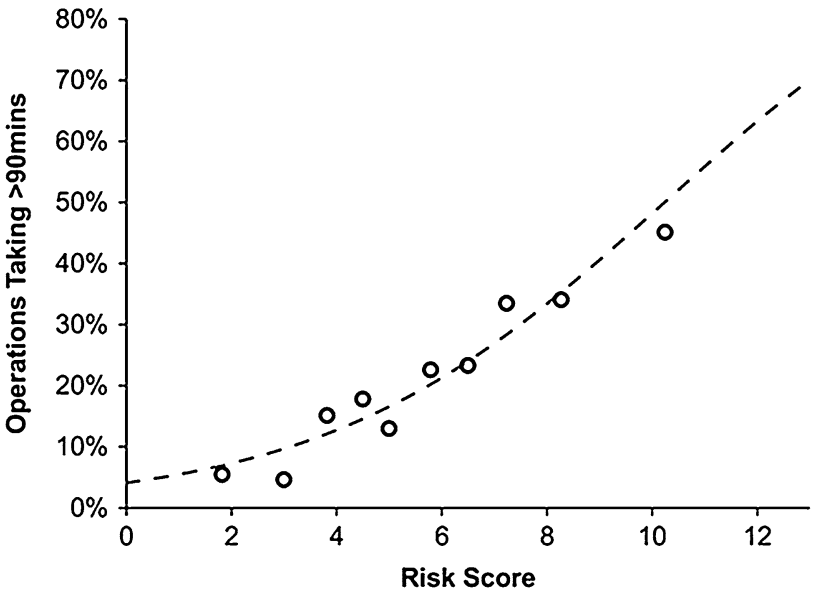

Fig. 1 Demonstrates the relationship between the risk score and the proportion of operations taking $>90 \mathrm{~min}$ in the validation cohort. Of the 470 patients with risk scores of $0-3$, only $5.1 \%(N=24)$ of operations took $>90$ min, increasing to $41.8 \%$ (109/261) in those with risk scores $>8$

possible, given that laparoscopic cholecystectomies are one of the most commonly performed operations in the NHS [1].

Thiels et al. [12] assessed the surgical duration of 1801 elective laparoscopic cholecystectomies from 2007 to 2013 and found female sex, BMI, ASA grade and pre-operative laboratory results to be predictive factors in influencing operative duration. They used a large group of patients from the NSQIP (American College of Surgeons National Surgical Quality Improvement Program) to validate their findings [12]. Zdichavsky et al. [20] performed a retrospective analysis of 677 consecutive patients undergoing laparoscopic cholecystectomies from 2004 to 2007 (excluding conversions, intra-operative cholangiogram and concurrent liver cirrhosis) and found male sex, obesity, acute cholecystitis and previous abdominal surgery to be independently predictive of duration. In a small study of only 138 cholecystectomies, junior residents took significantly longer to complete a cholecystectomy than their senior counterparts $(p<0.05)$ [21].

In our study, factors found to be independently predictive of operative duration were patient age, gender, ASA grade, operative indication, BMI, CBD diameter, gallbladder wall thickness, pre-operative CT scan, planned intra-operative cholangiogram and the number of previous surgical admissions. These factors are broadly similar to previous work by other researchers [12, 20,21]. Our study expands on their work and has developed a clinically useful scoring tool. To our knowledge, this is the first study to use patient factors to create a validated scoring tool to predict operative duration for elective laparoscopic cholecystectomies.

Our study uses high-quality, validated, prospective data that were collected as part of the CholeS study. The 
substantial cohort of 7227 patients is considerably larger than those used by past researchers, which altogether gives greater assurance as to the reliability of the derived scoring tool. In addition, we have external validated our scoring tool and its utility to successfully predict operative duration. Our scoring tool can be used pre-operatively and was developed in a dataset which included patients who underwent conversion to open surgery and cholangiography and is therefore more generalizable. There are however some limitations that should be considered when analysing this study's results. The CholeS study did not collect data on pre-operative blood results, such as white cell count or CRP, or whether the patient had previous Upper GI surgery, which may indicate difficult surgery [22]. We recognise that the validation dataset was retrospectively collected from routine hospital data and therefore may have some inaccuracies. ASA grades were calculated retrospectively based on information from the patients' clinical records. However, even when ASA is calculated by anaesthetists there is an element of bias and variation [23]. Furthermore, this scoring tool has been developed for elective cholecystectomy data and should not be used to predict the duration of acute operations.

\section{Conclusion}

We have created a scoring tool to predict operative durations of elective laparoscopic cholecystectomies using preoperative patient factors. Whilst previous research may have examined the significance of individual factors, there remained a lack of a formal scoring tool. Using the 7227 patient CholeS dataset to derive the scoring system, and a UHB database of 2405 patients to subsequently validate the tool, we have shown that it is possible to predict operations that are likely to last greater than $90 \mathrm{~min}$. This could be useful for theatre schedulers to ensure theatre lists are planned appropriately to optimise theatre utilisation and achieve cost savings. For example, the tool could be used to select the most appropriate patients to fit into a half day operating list with a low risk of overrunning. Another use could be to place patients with a long operative duration (and hence higher operative difficultly) on a specialist surgeon's operating list.

Acknowledgements We would like to acknowledge the help of Amanda Kirkham, Statistician, Birmingham Clinical Trials Unit for help with managing the CholeS database, and Suwathini Ravichandran, Birmingham Health Informatics, for help with the UHB database.

\section{CholeS Study Management Team}

Ravinder S. Vohra, Consultant Surgeon, Nottingham OesophagoGastric Unit, Nottingham University Hospitals NHS Foundation Trust, Hucknall Road, Nottingham, UK;

Amanda J. Kirkham, Biostatistician; Cancer Research UK Clinical Trials Unit, The University of Birmingham, Birmingham, UK;
Sandro Pasquali, Surgical trainee, Surgical Oncology Unit, Veneto Institute of Oncology IOV-IRCCS, Padova, Italy;

Paul Marriott, Surgical trainee, West Midlands Research Collaborative, Academic Department of Surgery, The University of Birmingham, Birmingham, UK;

Marianne Johnstone, Surgical trainee, West Midlands Research Collaborative, Academic Department of Surgery, The University of Birmingham, Birmingham, UK;

Philip Spreadborough, Surgical trainee, West Midlands Research Collaborative, Academic Department of Surgery, The University of Birmingham, Birmingham, UK

Derek Alderson, Emeritus Professor of Surgery, Academic Department of Surgery, The University of Birmingham, Birmingham, UK;

Ewen A. Griffiths, Consultant Surgeon, Department of Upper Gastrointestinal Surgery, University Hospitals Birmingham NHS Foundation Trust, Birmingham, UK

CholeS Study Collaborators:

England-Stephen Fenwick, Mohamed Elmasry, Quentin M. Nunes, David Kennedy (Aintree University Hospital NHS Foundation Trust); Raja Basit Khan, Muhammad A. S. Khan (Airedale General Hospital); Conor J. Magee, Steven M. Jones, Denise Mason, Ciny P. Parappally (Wirral University Teaching Hospital); Pawan Mathur, Michael Saunders, Sara Jamel, Samer Ul Haque, Sara Zafar (Barnet and Chase Farm Hospital); Muhammad Hanif Shiwani, Nehemiah Samuel, Farooq Dar, Andrew Jackson (Barnsley District General Hospital); Bryony Lovett, Shiva Dindyal, Hannah Winter, Ted Fletcher, Saquib Rahman (Basildon Univesity Hospital); Kevin Wheatley, Tom Nieto, Soofiyah Ayaani (Sandwell and West Birmingham Hospitals NHS Trust); Haney Youssef, Rajwinder S. Nijjar, Helen Watkin, David Naumann, Sophie Emesih; Piyush B. Sarmah, Kathryn Lee, Nikita Joji, Joel Lambert (Heart of England Foundation NHS Trust); Jonathan Heath, Rebecca L. Teasdale, Chamindri Weerasinghe (Blackpool Teaching Hospitals NHS Foundation Trust); Paul J. Needham, Hannah Welbourn, Luke Forster, David Finch (Bradford Teaching Hospitals NHS Foundation Trust); Jane M. Blazeby, William Robb, Angus G. K. McNair, Alex Hrycaiczuk (University Hospitals Bristol NHS Trust); Alexandros Charalabopoulos, Sritharan Kadirkamanathan, CheukBong Tang, Naga V. G. Jayanthi, Nigel Noor (Broomfield Hospital); Brian Dobbins, Andrew J. Cockbain, April Nilsen-Nunn, Jonathan de Siqueira (Calderdale and Huddersfield NHS Trust); Mike Pellen, Jonathan B. Cowley, Wei-Min Ho, Victor Miu (Hull and East Yorkshire NHS Trust); Timothy J. White, Kathryn A. Hodgkins, Alison Kinghorn (Chesterfield Royal Hospital NHS Foundation Trust); Matthew G. Tutton, Yahya A. Al-Abed, Donald Menzies, Anwar Ahmad, Joanna Reed, Shabuddin Khan (Colchester Hospital University NHS Foundation Trust); David Monk, Louis J. Vitone, Ghulam Murtaza, Abraham Joel (Countess of Chester NHS Foundation Trust); Stephen Brennan, David Shier, Catherine Zhang, Thusidaran Yoganathan (Croydon Health Services NHS Trust); Steven J. Robinson, Iain J. D. McCallum, Michael J. Jones, Mohammed Elsayed, Liz Tuck, John Wayman, Kate Carney (North Cumbria University Hospitals Trust); Somaiah Aroori, Kenneth B. Hosie, Adam Kimble, David M. Bunting, Kenneth B. Hosie (Plymouth Hospitals NHS Trust); Adeshina S. Fawole, Mohammed Basheer, Rajiv V. Dave, Janahan Sarveswaran, Elinor Jones, Chris Kendal (Mid Yorkshire NHS Trust); Michael P. Tilston, Martin Gough, Tom Wallace, Shailendra Singh, Justine Downing Katherine A. Mockford, Eyad Issa, Nayab Shah, Neal Chauhan (Northern Lincolnshire and Goole NHS Foundation Trust); Timothy R. Wilson, Amir Forouzanfar, Jonathan R. L. Wild, Emma Nofal, Catherine Bunnell, Khaliel Madbak (Doncaster and Bassetlaw Hospitals NHS Foundation Trust); Sudhindra T. V. Rao, Laurence Devoto, Najaf Siddiqi, Zechan Khawaja (Dorset County Hospital NHS Foundation Trust); James C. Hewes, Laura Gould, Alice Chambers, Daniel Urriza Rodriguez (North Bristol NHS Trust); Gourab Sen, Stuart Robinson, Kate Carney, Francis Bartlett (Freeman Hospital); David M. Rae, Thomas E. J. Stevenson, Kas Sarvananthan (Frimley Park Hospital NHS Trust); Simon J. Dwerryhouse, 
Simon M. Higgs, Oliver J. Old, Thomas J. Hardy, Reena Shah Steve T. Hornby, Ken Keogh, Lucinda Frank (Gloucestershire Hospitals NHS Trust); Musallam Al-Akash, Emma A. Upchurch (Great Western Hospitals NHS Foundation Trust); Richard J. Frame, Michael Hughes, Clare Jelley (Harrogate and District NHS Foundation Trust); Simon Weaver, Sudipta Roy, Toritseju O. Sillo, Giorgios Galanopoulos (Wye Valley NHS Trust); Tamzin Cuming, Pedro Cunha, Salim Tayeh, Sarantos Kaptanis (Homerton University Hospital NHS Trust); Mohamed Heshaishi, Abdalla Eisawi, Michael Abayomi; Wee Sing Ngu, Katie Fleming, Dalvir S. Bajwa (Tees Hospitals NHS Foundation Trust); Vivek Chitre, Kamal Aryal, Paul Ferris (Paget University Hospitals NHS Foundation Trust); Michael Silva, Simon Lammy Sarah Mohamed, Amir Khawaja, Adnan Hussain, Mudassar A. Ghazanfar, Maria Irene Bellini (Oxford University NHS Trust); Hamdi Ebdewi, Mohamed Elshaer, Gianpiero Gravante, Benjamin Drake (Kettering General Hospital NHS Foundation Trust); Arikoge Ogedegbe, Dipankar Mukherjee, Chanpreet Arhi, Lola Giwa Nusrat Iqbal (Barking, Havering and Redbridge University Hospitals NHS Trust); Nicholas F. Watson, Smeer Kumar Aggarwal, Philippa Orchard, Eduardo Villatoro (Kings Mill Hospital); Peter D. Willson, Kam Wa Jessica Mok, Thomas Woodman, Jean Deguara (Kingston Hospital NHS Foundation Trust); Giuseppe Garcea, Benoy I. Babu, A. R. Dennison, Deep Malde, David Lloyd, Steve Satheesan, Omer Al-Taan, Alexander Boddy (University Hospitals of Leicester NHS Trust); John P. Slavin, Robert P. Jones, Laura Ballance, Stratos Gerakopoulos (Leighton Hospital, Mid Cheshire Hospitals NHS Foundation Trust); Periyathambi Jambulingam, Sami Mansour, Naomi Sakai, Vikas Acharya (Luton \& Dunstable University Hospital NHS Foundation Trust); Mohammed M. Sadat, Lawen Karim, David Larkin, Khalid Amin (Macclesfield District General Hospital); Amarah Khan, Jennifer Law, Saurabh Jamdar, Stella R. Smith, Keerthika Sampat, Kathryn M. O'shea (Central Manchester NHS Foundation Trust); Mangta Manu, Fotini M. Asprou, Nabeela S. Malik, Jessica Chang, Marianne Johnstone (Royal Wolverhampton Hospitals NHS Trust); Michael Lewis, Geoffrey P. Roberts, Babu Karavadra, Evangelos Photi (Norfolk and Norwich University Hospitals NHS Foundation Trust); James Hewes, Laura Gould, Alice Chambers, Dan Rodriguez (North Bristol NHS Trust); Derek A. O'Reilly, Anthony J. Rate, Hema Sekhar, Lucy T. Henderson, Benjamin Z. Starmer, Peter O. Coe, Sotonye Tolofari, Jenifer Barrie (Pennine Acute NHS Trust); Gareth Bashir, Jake Sloane, Suroosh Madanipour, Constantine Halkias, Alexander E. J. Trevatt (North Middlesex Trust); David W. Borowski, Jane Hornsby, Michael J. Courtney, Suvi Virupaksha (North Tees and Hartlepool NHS Foundation Trust); Keith Seymour, Sarah Robinson, Helen Hawkins, Sadiq Bawa, Paul V. Gallagher, Alistair Reid, Peter Wood (Northumbria Healthcare NHS Foundation Trust); J. G. Finch, J. Guy Finch, J. Parmar, E. Stirland (Northampton General Hospital NHS Trust); James Gardner-Thorpe, Ahmed AlMuhktar, Mark Peterson, Ali Majeed (Sheffield Teaching Hospitals NHS Foundation Trust); Farrukh M. Bajwa, Jack Martin, Alfred Choy, Andrew Tsang (Peterborough City Hospital); Naresh Pore, David R. Andrew, Waleed Al-Khyatt, Christopher Taylor, Santosh Bhandari, Adam Chambers, Dhivya Subramanium (United Lincolnshire Hospitals NHS Trust); Simon K. C. Toh, Nicholas C. Carter, Sophie Tate, Belinda Pearce, Denise Wainwright, Stuart J. Mercer, Benjamin Knight (Portsmouth Hospitals NHS Trust); Vardhini Vijay, Swethan Alagaratnam, Sidhartha Sinha, Shahab Khan (The Princess Alexandra Hospital NHS Trust); Shamsi S. El-Hasani, Abdulzahra A. Hussain (Kings College Hospital NHS Foundation Trust); Vish Bhattacharya, Nisheeth Kansal, Tani Fasih, Claire Jackson (Gateshead Health NHS Foundation Trust); Midhat N. Siddiqui, Imran A. Chishti, Imogen J. Fordham, Zohaib Siddiqui (Lewisham and Greenwich NHS Trust); Harald Bausbacher, Ileana Geogloma, Kabita Gurung (Queen Elizabeth Hospital NHS Trust); George Tsavellas, Pradeep Basynat, Ashish Kiran Shrestha, Sanjoy Basu, Alok Chhabra Mohan Harilingam, Mohamed Rabie, Mansoor Akhtar (East Kent Hospitals University NHS Foundation Trust); Pradeep Kumar, Sadaf F. Jafferbhoy, Najam Hussain,
Soulat Raza (Burton Hospitals NHS Foundation Trust); Manzarul Haque, Imran Alam, Rabiya Aseem, Shakira Patel, Mehek Asad (Royal Albert Edward Infirmary, Wigan Wrightington and Leigh NHS Trust); Michael I. Booth, William R. Ball, Christopher P. J. Wood, Ana C. Pinho-Gomes (Royal Berkshire NHS Foundation Trust); Ambareen Kausar, Moh'd Rami Obeidallah (East Lancashire Hospital Trust); Joseph Varghase, Joshil Lodhia, Donal Bradley, Carla Rengifo, David Lindsay (Royal Bolton Hospital NHS Foundation Trust); Sivakumar Gopalswamy, Ian Finlay, Stacy Wardle, Naomi Bullen (Royal Cornwall NHS Trust); Syed Yusuf Iftikhar, Altaf Awan, Javed Ahmed, Paul Leeder (Royal Derby NHS Foundation Trust); Guiseppe Fusai, Giles Bond-Smith, Alicja Psica, Yogesh Puri (Royal Free, London); David Hou, Fergus Noble, Karoly Szentpali, Jack Broadhurst (Hampshire Hospital NHS Foundation Trust); Ravindra Date, Martin R. Hossack, Yan Li Goh, Paul Turner, Vinutha Shetty (Lancashire Teaching HospitalsNHS Foundation Trust); Manel Riera, Christina A. W. Macano, Anisha Sukha (Royal Shrewsbury Hospital); Shaun R. Preston, Jennifer R. Hoban, Daniel J. Puntis, Sophie V. Williams (Royal Surrey County Hospital NHS Foundation Trust); Richard Krysztopik, James Kynaston, Jeremy Batt, Matthew Doe (Royal United Hospital Bath NHS Trust); Andrzej Goscimski, Gareth H. Jones, Stella R. Smith, Claire Hall (Salford Royal NHS Foundation Trust); Nick Carty, Jamil Ahmed, Sofoklis Panteleimonitis (Salisbury Hospital Foundation Trust); Rohan T. Gunasekera, Andrea R. G. Sheel, Hannah Lennon, Caroline Hindley (Southport and Ormskirk Hospital NHS Trust); Marcus Reddy, Ross Kenny, Natalie Elkheir, Emma R. McGlone (St George's Healthcare NHS Trust); Rajasundaram Rajaganeshan, Kate Hancorn, Anita Hargreaves (St Helens and Knowsley Teaching Hospitals NHS Trust); Raj Prasad, David A. Longbotham, Dhakshinamoorthy Vijayanand, Imeshi Wijetunga (Leeds Teaching Hospitals); Paul Ziprin, Christopher R. Nicolay, Geoffrey Yeldham, Edward Read (Imperial College Healthcare NHS Trust); James A. Gossage, Rachel C. Rolph, Husam Ebied, Manraj Phull (St Thomas' Hospital, London); Mohammad A. Khan, Matthew Popplewell, Dimitrios Kyriakidis, Anwar Hussain (Mid Staffordshire NHS Foundation Trust); Natasha Henley, Jessica R. Packer, Laura Derbyshire, Jonathan Porter (Stockport NHS Foundation Trust); Shaun Appleton, Marwan Farouk, Melvinder Basra (Bucks Healthcare NHS Trust); Neil A. Jennings, Shahda Ali, Venkatesh Kanakala (City Hospitals Sunderland NHS Foundation Trust); Haythem Ali, Risha Lane, Richard Dickson-Lowe, Prizzi Zarsadias (Tunbridge Wells and Maidstone NHS Trust); Darius Mirza, Sonia Puig, Khalid Al Amari, Deepak Vijayan, Robert Sutcliffe, Ravi Marudanayagam (University Hospital Birmingham NHS Foundation Trust); Zayed Hamady, Abheesh R. Prasad, Abhilasha Patel (University Hospital Coventry and Warwickshire NHS Trust); Damien Durkin, Parminder Kaur, Laura Bowen (University Hospital of North Staffordshire NHS Trust); James P. Byrne, Katherine L. Pearson, Theo G. Delisle, James Davies (University Hospital Southampton NHS Foundation Trust); Mark A. Tomlinson, Michelle A. Johnpulle, Corinna Slawinski (University Hospitals of Morecambe Bay); Andrew Macdonald, James Nicholson, Katy Newton, James Mbuvi (University Hospital South Manchester NHS Foundation Trust); Ansar Farooq, Bhavani Sidhartha Mothe, Zakhi Zafrani, Daniel Brett (Warrington and Halton Hospitals NHS Trust); James Francombe, Philip Spreadborough, James Barnes, Melanie Cheung (South Warwickshire NHS Foundation Trust); Ahmed Z. AlBahrani, Giuseppe Preziosi, Tomas Urbonas (Watford General Hospital); Justin Alberts, Mekhlola Mallik, Krashna Patel, Ashvina Segaran, Triantafyllos Doulias (West Suffolk NHS Trust); Pratik A. Sufi, Caroline Yao, Sarah Pollock (Whittington NHS Trust); Antonio Manzelli, Saj Wajed, Michail Kourkulos, Roberto Pezzuto (Wonford Hospital); Martin Wadley, Emma Hamilton, Shameen Jaunoo, Robert Padwick (Worcestershire Acute Hospitals NHS Trust); Mazin Sayegh, Richard C. Newton, Madhusoodhana Hebbar, Sameh F. Farag, (Western Sussex Hospitals NHS Foundation Trust); John Spearman, Mohammed F. Hamdan, Conrad D'Costa, Christine Blane; (Yeovil District Hospital NHS Trust); Mathew Giles, Mark B. Peter, Natalie A. Hirst, Tanvir 
Hossain, Arslan Pannu Yesar El-Dhuwaib, Tamsin E. M. Morrison, Greg W. Taylor (York Teaching Hospital NHS Foundation Trust).

Northern Ireland-Ronald L. E. Thompson, Ken McCune, Paula Loughlin, Roger Lawther (Altnagelvin Area Hospital); Colman K. Byrnes, Duncan J. Simpson, Abi Mawhinney, Conor Warren (Antrim Area Hospital); Damian McKay, Colin McIlmunn, Serena Martin, Matthew MacArtney (Daisy Hill Hospital); Tom Diamond, Phil Davey, Claire Jones, Joshua M. Clements, Ruairi Digney, Wei Ming Chan, Stephen McCain, Sadaf Gull, Adam Janeczko, Emmet Dorrian, Andrew Harris, Suzanne Dawson, Dorothy Johnston, Barry McAree, (Belfast City Hospital, Mater Infirmorum Hospital Belfast and Royal Victoria Hospital); Essam Ghareeb, George Thomas, Martin Connelly, Stephen McKenzie, Krzysztos Cieplucha (South West Acute Hospital); Gary Spence, William Campbell, Gareth Hooks, Neil Bradley (Ulster Hospital).

Republic of Ireland-Arnold D. K. Hill, John T. Cassidy, Michael Boland (Beaumont Hospital, Dublin); Paul Burke, Deirdre M. Nally (University Hospital Limerick); Arnold D. K. Hill, Elmoataz Khogali, Wael Shabo, Edrin Iskandar (Louth County Hospital and Our Lady of Lourdes Hospital); Gerry P. McEntee, Maeve A. O’Neill, Colin Peiree, Emma M. Lyons (Mater Hospital, Dublin); Adrian W. O’Sullivan, Rohan Thakkar, Paul Carroll, Ivan Ivanovski (Mercy University Hospital); Paul Balfe, Matthew Lee (St Luke's General Hospital Kilkenny); Des C. Winter, Michael E. Kelly, Emir Hoti, Donal Maguire; Priyadarssini Karunakaran, Justin G. Geoghegan, Frank McDermott, Sean T. Martin (St Vincent's University and Private Hospitals, Dublin); Keith S. Cross, Fiachra Cooke, Saquib Zeeshan, James O. Murphy (Waterford Regional Hospital); Ken Mealy, Helen M. Mohan, Yuwaraja Nedujchelyn, Muhammad Fahad Ullah (Wexford General Hospital).

Scotland-Irfan Ahmed, Francesco Giovinazzo, James Milburn (Aberdeen Royal Infirmary); Sarah Prince, Eleanor Brooke, Joanna Buchan (Belford Hospital); Ahmed M. Khalil, Elizabeth M. Vaughan, Michael I. Ramage, Roland C. Aldridge (Borders General Hospital); Simon Gibson, Gary A. Nicholson, David G. Vass (Crosshouse Hospital, Ayrshire \& Arran); Alan J. Grant, David J. Holroyd, M. Angharad Jones, Cherith MLR Sutton (Dr Gray's Hospital); Patrick O'Dwyer, Frida Nilsson (Gartnavel General Hospital); Beatrix Weber, Tracey K. Williamson, Kushik Lalla, Alice Bryant (Gilbert Bain Hospital); C. Ross Carter, Craig R. Forrest, David I. Hunter (Glasgow Royal Infirmary); Ahmad H. Nassar, Mavis N. Orizu, Katrina Knight, Haitham Qandeel (Monklands Hospital); Stuart Suttie, Rowena Belding, Andrew McClarey (Ninewells Hospital); Alan T. Boyd, Graeme J. K. Guthrie, Pei J. Lim, Andreas Luhmann (Perth Royal Infirmary); Angus J. M. Watson, Colin H. Richards, Laura Nicol, Marta Madurska (Raigmore Hospital); Ewen Harrison, Kathryn M. Boyce, Amanda Roebuck, Graeme Ferguson (Royal Infirmary of Edinburgh); Pradeep Pati, Michael S. J. Wilson, Faith Dalgaty, Laura Fothergill (Stracathro Hospital); Peter J. Driscoll, Kirsty L. Mozolowski, Victoria Banwell, Stephen P. Bennett (Victoria Hospital, Kirkcaldy); Paul N. Rogers, Brendan L. Skelly, Claire L. Rutherford, Ahmed K. Mirza (Western Infirmary Glasgow).

Wales-Taha Lazim, Henry C. C. Lim, Diana Duke, Talat Ahmed (Bronglais General Hospital); William D. Beasley, Marc D. Wilkinson, Geta Maharaj, Cathy Malcolm (Glangwili General and Prince Philip Hospital); Timothy H. Brown, Bilal Al-Sarireh, Guy M. Shingler, Nicholas Mowbray, Rami Radwan (Morriston and Singleton Hospitals); Paul Morcous, Simon Wood, Abbas Kadhim (Princess of Wales Hospital); Duncan J. Stewart, Andrew L. Baker, Nicola Tanner, Hrishikesh Shenoy (Wrexham Maelor Hospital).

Data validators-Shazia Hafiz, Joshua A. De Marchi, Deepak Singh-Ranger, Elzanati Hisham, Paul Ainley, Stephen O’Neill, John Terrace, Sara Napetti, Benjamin Hopwood, Thomas Rhys, Justine Downing, Sam Kanavati, Maria Coats, Danail Aleksandrov, Charlotte Kallaway, Salama Yahya, Beatrix Weber, Alexa Templeton, Martin Trotter, Christina Lo, Ajit Dhillon, Nick Heywood, Yousif Aawsaj, Alhafidz Hamdan, Obuobi Reece-Bolton, Andrew McGuigan, Yousef
Shahin, Aymon, Ali Alison Luther, James A. Nicholson, Ilayaraja Rajendran, Matthew Boal, Judith Ritchie.

\section{Compliance with ethical standards}

Disclosure Miss Reshma Bharamgoudar, Mr Aniket Sonsale, Mr James Hodson and Mr Ewen Griffiths have no conflicts of interest or financial ties to disclose.

Open Access This article is distributed under the terms of the Creative Commons Attribution 4.0 International License (http://creativecommons.org/licenses/by/4.0/), which permits unrestricted use, distribution, and reproduction in any medium, provided you give appropriate credit to the original author(s) and the source, provide a link to the Creative Commons license, and indicate if changes were made.

\section{References}

1. NHS Digital (2017) Hospital admitted patient care activity, pp 2015-2016

2. Subhas G, Gupta A, Bhullar J, Dubay L, Ferguson L, Goriel Y, Jacobs MJ, Kolachalam RB, Silapaswan S, Mittal VK (2011) Prolonged (longer than 3 hours) laparoscopic cholecystectomy: reasons and results. Am Surg 77:981-984

3. NHS (2009) Improving quality and efficiency in the operating theatre. NHS Institute for Innovation and Improvement, London

4. Carter P (2016) Operational productivity and performance in English NHS acute hospitals: Unwarranted variations. Department of Health, London

5. Kumar R, Gandhi R (2012) Reasons for cancellation of operation on the day of intended surgery in a multidisciplinary 500 bedded hospital. J Anaesthesiol Clin Pharmacol 28:66-69

6. Dexter F, Macario A, Epstein RH, Ledolter J (2005) Validity and usefulness of a method to monitor surgical services' average bias in scheduled case durations. Can J Anaesth 52:935-939

7. Dexter F, Dexter EU, Masursky D, Nussmeier NA (2008) Systematic review of general thoracic surgery articles to identify predictors of operating room case durations. Anesth Analg 106:12321241 (table of contents)

8. Eijkemans MJ, van Houdenhoven M, Nguyen T, Boersma E, Steyerberg EW, Kazemier G (2010) Predicting the unpredictable: a new prediction model for operating room times using individual characteristics and the surgeon's estimate. Anesthesiology 112:41-49

9. Gambadauro P, Campo V, Campo S (2015) How predictable is the operative time of laparoscopic surgery for ovarian endometrioma? Minim Invasive Surg 2015:702631

10. Hosseini N, Hallbeck MS, Jankowski CJ, Huddleston JM, Kanwar A, Pasupathy KS (2014) Effect of obesity and clinical factors on pre-incision time: study of operating room workflow. AMIA Ann Symp Proc 2014:691-699

11. Walter SD (1973) A comparison of appointment schedules in a hospital radiology department. Br J Prev Soc Med 27:160-167

12. Thiels CA, Yu D, Abdelrahman AM, Habermann EB, Hallbeck S, Pasupathy KS, Bingener J (2017) The use of patient factors to improve the prediction of operative duration using laparoscopic cholecystectomy. Surg Endosc 31:333-340

13. CholeS Study Group WMRC (2016) Population-based cohort study of outcomes following cholecystectomy for benign gallbladder diseases. Br J Surg 103:1704-1715 
14. CholeS Study Group WMRC (2016) Population-based cohort study of variation in the use of emergency cholecystectomy for benign gallbladder diseases. Br J Surg 103:1716-1726

15. Vohra RS, Spreadborough P, Johnstone M, Marriott P, Bhangu A, Alderson D, Morton DG, Griffiths EA, West Midlands Research Collaborative (2015) Protocol for a multicentre, prospective, population-based cohort study of variation in practice of cholecystectomy and surgical outcomes (The CholeS study). BMJ Open 5:e006399

16. University Hospitals Birmingham NHS Foundation Trust (2017) About us. University Hospitals Birmingham NHS Foundation Trust, Birmingham

17. American Society of Anaesthesiologists (2017) ASA physical status classification system. American Society of Anaesthesiologists, Schaumburg

18. Archer T, Macario A (2006) The drive for operating room efficiency will increase quality of patient care. Curr Opin Anaesthesiol 19:171-176
19. Health.org.uk (2017) 6 in 10 NHS trusts in deficit Health.org

20. Zdichavsky M, Bashin YA, Blumenstock G, Zieker D, Meile T, Konigsrainer A (2012) Impact of risk factors for prolonged operative time in laparoscopic cholecystectomy. Eur J Gastroenterol Hepatol 24:1033-1038

21. Souadka A, Naya MS, Serji B, El Malki HO, Mohsine R, Ifrine L, Belkouchi A, Benkabbou A (2017) Impact of seniority on operative time and short-term outcome in laparoscopic cholecystectomy: experience of an academic Surgical Department in a developing country. J Minim Access Surg 13:131-134

22. Goh YL, Toumi Z, Mok KW, Date RS (2015) Variation in conversion rates of laparoscopic cholecystectomy in different set-ups can be explained on the basis of C-reactive protein (CRP) values at index admission. Int J Surg 15:38-39

23. Sankar A, Johnson SR, Beattie WS, Tait G, Wijeysundera DN (2014) Reliability of the American Society of Anesthesiologists physical status scale in clinical practice. Br J Anaesth 113:424-432 